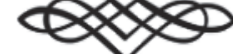

Hermeneutical Bioethics: a critical review of principialist approach

\title{
Bioética hermenéutica: una revisión crítica del enfoque principialista
}

Sergio Quintero Martín

Universidad Complutense de Madrid

quinte2002@hotmail.com

DOI: http://dx.doi.org/10.15366/bp2018.18.009

Bajo Palabra. II Época. No18. Pgs: 195-208

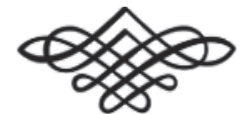


Recibido: 10/07/2017

Aprobado: 29/07/2018

\section{Resumen}

El propósito de este artículo es intentar mostrar las progresivas carencias, tanto a nivel teórico como, sobre todo, a nivel práctico, de la metodología del enfoque principialista para el análisis ético de los problemas que surgen diariamente en la práctica médica. La cuestión es: ¿los conflictos éticos actualmente son el resultado de una mala gestión y una mala perspectiva institucional?, ¿son algo inevitable en la práctica médica?, ¿๐ son ambas?

Palabras clave: enfoque principialis$t a$, conflicto ético, hermenéutica, análisis bioético.

\section{Abstract}

The goal of this article is try to explain the progressive deficiencies, both theoretically and, above all, at the practical level, of the methodology of the principialist approach for the ethical analysis of the problems that arise daily in medical practice. The question is: are the ethical conflicts currently the result of poor management and poor institutional perspective? Are they inevitable in medical practice? Or are both?.

Keywords: principialist approach, ethical conflict, hermeneutic, bioethical analysis. 


\section{Una duda razonable}

Los CONFLICTOS ÉTICOS SON LA MONEDA DE LA BioÉTiCA médica. ${ }^{1}$ En otras palabras, el fundamento de la bioética son los conflictos éticos, es decir, el aspecto práctico (no-técnico) de la práctica médica. Desoír la causa constitutiva de la bioética es juzgar un problema despojado de un sentido hermenéutico, validando sus consecuencias como infructuosas por defecto o volviéndolas estériles por exceso.

El análisis de los conflictos éticos existentes en la práctica médica se lleva a cabo metodológicamente desde la aparición del Informe Belmont (1979). En este momento, nacieron simultáneamente dos aproximaciones para lidiar con estas cuestiones que, si bien no son radicalmente contrapuestas, tienen ciertos elementos divergentes: por un lado, el enfoque principialista, y, por otro lado, el enfoque casuístico. La primera aproximación fue desarrollada por Tom Beauchamp y Jamen Franklin Childress y se caracteriza por "la combinación de unos principios abstractos con unas reglas de mediación que servirán como estrategia práctica” ${ }^{2}$ frente a los conflictos éticos. La segunda aproximación adoptada principalmente por Albert Jonsen y Stephen Toulmin se basa en la primacía del caso particular en torno al que se dirime una solución ética y biomédica a través de juicios contingentes y hermenéuticos. De ambos enfoques, el adoptado de forma institucional por la mayoría de los sistemas sanitarios fue el enfoque principialista porque ofrece un marco general que elimina la arbitrariedad de la toma de decisiones y un fundamento asumible como universal, frente a la heterogeneidad y la aparente falta de fundamentación del enfoque casuístico.

Si bien es cierto que a través del marco general que establece el enfoque principialista se alcanzó una primera solución a problemas éticos surgidos desde mediados del siglo pasado como la conjugación de los rápidos avances tecnológicos con unos efectos inciertos, la protección frente a atrocidades similares a las del programa de la medicina nazi, ${ }^{3}$ los nuevos métodos de tratamiento que resultaron tremenda-

\footnotetext{
${ }^{1}$ Parafraseando el inicio de un artículo de Albert Jonsen donde aborda el interés del enfoque casuístico como metodología para la ética médica.

Véase, Jonsen, Albert. "Casuistry as Methodology in Clinical Ethics", en Theoretical Medicine, 12, 1991, pp. 299-302.

2 Domingo Moratalla, Tomás; y Feito Grande, Lydia. Bioética narrativa, Madrid, Escolar y Mayo Editores, 2013, p. 85.

3 Como ocurrió en los Estados Unidos entre las décadas de cuarenta y los sesenta, daremos tres ejemplos: en los ańos cuarenta en Alabama, seiscientos enfermos sifilíticos de raza negra fueron reclutados para un estudio sobre la evolución natural de la enfermedad, evitando tratarles (aun conociendo los efectos curativos de la penicilina
} 
mente invasivos, la jerarquización de las relaciones sanitarias, y el intento de construir un modelo sanitario universal de calidad (gracias al surgimiento generalizado en Europa del llamado Estado del Bienestar); hay que destacar que se trata de soluciones reducidas a problemas técnicos y tecnológicos. No negamos que el enfoque principialista no funcione y deba desecharse, todo lo contrario: los problemas que originaron su institución han sido resueltos con bastante acierto. Sin embargo, se trata de una aproximación insuficiente ante la complejidad y la multidimensionalidad de los problemas bioéticos de la actualidad.

Susan Wolf ${ }^{4}$ describe una primera duda razonable sobre el empleo contemporáneo del marco general del enfoque principialista criticando su "culpable ceguera» y la inocencia formal a la hora de responder ante ciertos conflictos éticos de la medicina:

Frente al modelo de principios, que es el que parece predominante, se plantea la posibilidad de que la atención a lo general esté obstaculizando la visión de las diferencias y los factores contextuales, de que la insistencia en la autonomía se asiente sobre un modelo de individualismo liberal, poco sensible a las relaciones intersubjetivas y a la interdependencia entre las personas. ${ }^{5}$

Esta duda razonable sobre la pertinencia del enfoque principialista tiene como núcleo lo que Henk Ten Have califica como "paradoja bioética": "intentamos tratar los asuntos morales de la medicina con una concepción de la ética que en sí misma está impregnada de racionalidad científico-técnica" . ${ }^{6}$ En otras palabras, la "paradoja bioética” representa la contradicción que surge al primar la racionalidad técnica sobre el contexto en los procedimientos médicos. De este modo, en la práctica médica la validez de los protocolos y de los procedimientos son de facto incuestionables (pese a que teóricamente en el enfoque principialista hay margen a la corrección y la autocrítica), y su único objetivo es el de clarificar responsabilidades de las acciones, es decir, como se dice coloquialmente, "lavarse las manos" frente a cualquier fallo procedimental o protocolario: la culpa fue del paciente que no entendió el consen-

sobre la sífilis) y realizando las autopsias a los paciencias fallecidos; entre los años 1959 y 1962 nacieron más de ocho mil niños con deformaciones congénitas severas debido a la ingesta por parte de las madres -durante el período de gestación- de la Thalidomida (un fármaco creado para reducir y aliviar las consecuencias del embarazo, como las náuseas); y, por último, en 1963, en un hospital de Brooklyn (Nueva York), se inyectaron células cancerosas vivas a un grupo de ancianos, y poco tiempo después, en un hospital-también neoyorkino-se inoculó el virus de la hepatitis en una serie de niños con minusvalías psíquicas.

4 Véase, Wolf, Susan. "Gender and feminism in bioethics", en Feminism and Bioethics: beyond reproduction (Ed. Susan Wolf), Nueva York, Oxford University Press, 1996, pp. 3-43.

5 Domingo Moratalla, Tomás; y Feito Grande, Lydia. Bioética narrativa, op. cit., p. 89.

${ }^{6}$ Ten Have, Henk. "Bioética: el arte de la interpretación y la deliberación", en Bioética: el estado de la cuestión (Eds. Lydia Feito, Diego Gracia, Miguel Sánchez), Madrid, Editorial Triacastela, 2011, p.170. 
timiento, la culpa fue del médico por excederse en sus obligaciones o no realizarlas conforme a los protocolos, la culpa fue de la institución sanitaria por no disponer de recursos o de una calidad en el servicio. La consecuencia de esta paradoja es que la práctica médica está guiada por el miedo al litigio, haciendo que la medicina sea una práctica que se despliega a medias porque se despliega a la defensiva.

El enfoque principialista se organiza, siguiendo una dialéctica medios-fines, como una construcción teórica sólida que, en sí misma, es acertada y coherente. Sin embargo, resulta ineficaz en un sentido práctico más allá de las cuestiones técnicas a las que se encuentra reducida, precisamente porque adolece de un despliegue perverso de sus principios. Este reduccionismo técnico se produce por el "fallo técnico" que surge en toda dialéctica medios-fines porque, como acentúa Antonio Gómez Ramos, "toda mecánica de medios y fines lleva aparejado el peligro de que los fines acaben sobrepasados, desvirtuados o anulados por los medios"7. La transvaloración del fin que pretende alcanzar la medicina -ayudada por la bioética- siguiendo el enfoque principialista (la curación de los seres humanos) se simplifica e instrumentaliza por su aspecto técnico (tecnologías más precisas y efectivas, técnicas quirúrgicas menos invasivas, fármacos más potentes). De esta manera, se identifica exclusivamente el fin de la medicina con ese medio particular y prescindiendo en el proceso del carácter biográfico, narrativo y contextual de la propia medicina.

El problema, por tanto, es que a raíz de esta paradoja se rompe la llamada "pax bioethica», ${ }^{8}$ lo que se agrava si tenemos en cuenta que la aproximación institucional a los conflictos ética se realiza siguiendo el enfoque principialista. "La bioética buscaba establecer un marco consensual de expertos y elaborar un aparato ético regulador autolegitimado. Se obviaron así las diferencias ideológicas y se buscaron grandes pactos tácitos para resolver los conflictos". ${ }^{9}$ La ruptura de la validez del enfoque principialista se debe a la dogmatización e instrumentalización de sus postulados para conjugar intereses exógenos a la medicina: intereses políticos e intereses económicos, subyacentes al sistema sanitario: privatización de servicios, intereses farmacéuticos, beneficios corporativos, etc. Esta ruptura de la «pax bioethica» transforma la bioética en una herramienta unidimensional cuya inocencia formal se encuentra al servicio de esos intereses técnicos y exógenos a la práctica médica.

\footnotetext{
7 Gómez Ramos, Antonio. "Política sin medios y violencia sin fines: Hannah Arendt y Walter Benjamin sobre la violencia”, en Cuaderno gris, No. 10, 2012, p. 187.

8 Moreno, Jonathan. "The end of the great bioethics compromise", en The Hastings Center Report, 35, 2005, pp. 14-15.

9 Domingo Moratalla, Tomás; y Feito Grande, Lydia. Bioética narrativa, op. cit., pp. 89-90.
} 


\section{El enfoque principialista: características y carencias.}

UNA VEZ EXPUESTA LA DUDA RAZONABLE sobre la pertinencia del enfoque principialista como filtro y baremo para el análisis metodológico de los conflictos éticos en la práctica médica, el siguiente paso es realizar un sucinto examen en torno a las principales características teóricas de este enfoque para mostrar que aunque provenga a la deliberación moral de una estructura sólida y coherente, el recurso a principios generales como elemento nuclear del análisis bioético resulta insuficiente para satisfacer la creciente complejidad ${ }^{10}$ de los conflictos éticos.

El enfoque principialista asume la necesidad de un fundamento universal para la ética (en este caso para bioética) para evitar que la deliberación y la consecuente toma de decisiones sea arbitraria o esté sometida a cuestiones coyunturales que los determinen. Por esta razón se establecen unos juicios apodícticos y a priori que garanticen la objetividad tanto de la deliberación como de la decisión basados en diferentes modelos de fundamentación moral (modelo deductivo, modelo inductivo, o modelo integrado) para después ser aplicados a cada caso particular dentro del contexto determinado. El objetivo es evitar un sistema ético absurdo y anárquico donde se puede legitimar cualquier tipo de decisión.

Para cumplir este objetivo se recurre a un sistema prescriptivo de principios prima facie que se encuentran a caballo en un nivel intermedio entre los principios morales absolutos (como podrían ser el imperativo categórico kantiano o el principios utilitarista milliano) y las reglas de acción, y, por tanto, resultan demasiado indeterminados para realizar una aplicación directa ${ }^{11}$ a los casos concretos de conflicto ético. Este sistema de principios prima facie está conformado por cuatro principios que se encontrarían en un mismo nivel horizontal de importancia sin que ninguno tenga una primacía de antemano. A través del estudio del caso concreto se revelará cuál tiene la primacía en el análisis bioético. Los principios que forman el canon del enfoque principialista son los siguientes:

${ }_{10}$ Esto no quiere decir que nos conflictos éticos no fueran complejos hasta sino que el análisis estaba restringido a una perspectiva unidimensional, suficiente para establecer un primer marco general para la bioética pero insatisfactorio a largo plazo, precisamente porque "la bioética ha ignorado las cuestiones culturales, socio-económicas y de género, olvidando por tanto, en la mayor parte de los casos, la pertenencia a una comunidad o a una sociedad, en la que la identidad de los individuos cobra sentido y en la que se pueden encontrar las eventuales raíces de situaciones de conflicto o injusticia” (Domingo Moratalla, Tomás; y Feito Grande, Lydia. Bioética narrativa, op. cit., p. 90).

11 Para poner en práctica en casos concretos el sistema indeterminado de principios prima facie se articulan diversas respuestas: el legalismo (aplicando los principios acorde con los mandatos de la ley), el intuicionismo (sin recurrir a una regla moral predefinida, simplemente apelando a un solucionismo contextual), y el situiacionismo (conjugando los principios con la experiencia moral de cada individuo). Si bien Beauchamp y Childress estarían próximos a esta última respuesta no se consideran de facto situacionistas. 
1. El principio de autonomía. Este principio aboga no sólo por la no-intervención "en los asuntos de otras personas, sino también la de asegurar las condiciones necesarias para que su elección sea autónoma, mitigando los miedos y todas aquellas circunstancias que puedan dificultar o impedir la autonomía del acto" ${ }^{12}$. Mediante el principio de respeto a la autonomía el paciente es considerado, en términos ideales, como un ente independiente y responsable capaz de tomar decisiones razonables y prudentes con la información suficiente, es decir, es un soberano de sí mismo competente para decidir sobre aquello que, médicamente, le afecta como paciente. La cristalización de este principio es el consentimiento informado que aporta al paciente la competencia, la capacidad, la revelación, la comprensión y la voluntariedad necesarias para la deliberación y la toma de decisiones autónomas.

2. El principio de beneficencia. Este principio se basa en la responsabilidad que contrae el médico con el paciente para buscar una curación y el restablecimiento de su salud, poniendo sus conocimientos y habilidades a su servicio. En otras palabras, es "la obligación moral de actuar en beneficio de otros". ${ }^{13}$

3. El principio de no-maleficencia. Este principio se puede entender de dos maneras: como un corolario que expresa de forma negativa y pasiva del principio de beneficencia, evitando realizar una acción aparentemente perjudicial; o como un principio activo en sí mismo basado en la expresión latina primum non nocere, es decir, como "la forma de no hacer daño intencionalmente". ${ }^{14}$ La obligación moral a la no-maleficencia implícita en la beneficencia de la práctica médica forman la base del corpus hipocraticum en el que se fundamenta la medicina clásica.

4. El principio de justicia. Este principio busca que dentro del ejercicio de la medicina cada paciente reciba un trato igualitario al de los demás sin importar los condicionamientos sociales externos: el color de piel, el credo, la sexualidad, el nivel económico, la cultura. El principio de justicia formal remite, en última instancia, a la noción de «justicia distributiva» de Aristóteles ${ }^{15}$ y su comprensión actual se basa en la noción sobre la justicia del jurista romano Domicio Ulpiano: Ius suum unicuique tribuens, es decir, los iguales deben ser tratados con igualdad. A partir de este principio se crea un marco ideal de imparcialidad para la práctica médica porque "no establece ámbitos concretos en los que los iguales deban ser tratados igualmente". ${ }^{16}$

\footnotetext{
12 Beauchamp, Tom; y Childress, James Franklin. Principios de ética biomédica, Barcelona, Editorial Masson, 1999, pp. 117-118.

13 Ibíd., p. 246.

14 Ibíd., p. 179.

15 Véase, Aristóteles. Ética Nicomáquea. Ética Eudemia, Madrid, Editorial Gredos, 2008.

16 Beauchamp, Tom; y Childress, James Franklin. Principios de ética biomédica, Barcelona, Editorial Masson, 1999, op. cit., p. 313.
} 
En conjunto, el sistema de los cuatro principios prima facie del enfoque principialista funciona, siguiendo a Javier Gafo, de la siguiente manera: "La beneficencia y la no-maleficencia llevan a una evaluación de los beneficios y los riesgos; la autonomía conduce a la percepción de si existe un verdadero consentimiento informado; la justicia lleva a una selección equitativa". ${ }^{17}$ De esta manera, el enfoque principialista tiene a su disposición una matriz teórica formal y coherente para deliberación moral.

La insuficiencia de este enfoque se produce a la hora de "aterrizar" el sistema de principios prima facie en la realidad de los conflictos éticos, debido a su propia constitución prima facie, es decir, a su indeterminación a la hora de realizar una aplicación a un caso conflictivo concreto. La puesta en práctica del enfoque principialista generaría una serie de carencias que, por un lado, transforma la percepción de la matriz teórica en una estructura formal inocente, creando el ambiente idóneo para la dogmatización de los principios prima facie; y, por otro lado, desequilibra la propia práctica bioética, pervirtiéndola.

De esta manera, y valiéndonos de algunas reflexiones de Diego Gracia en este punto, ${ }^{18}$ se produciría una carencia en la fundamentación del procedimiento práctico del razonamiento ético principialista. La forma de proceder a poner en práctica el sistema de principios prima facie se encuentra inserta en la dialéctica medios-fines que caracteriza a la razón formal y la metodología lineal propia de este enfoque, por lo que resulta inevitable su caída en el "fallo técnico" que comenta Antonio Gómez Ramos. A la hora de analizar el despliegue de los principios prima facie se pone en evidencia la inocencia formal del enfoque principialista: para solventar los problemas que produce la indeterminación de la naturaleza prima facie de los cuatro principios se realiza un recurso de petición de principios (petitio principii) apelando a principios anteriores, o principios meta-éticos, subjetivos que provocan la mediación de concepciones personalistas en la práctica bioética, rompiendo con la garantía de objetividad que promete el enfoque principialista.

La dogmatización de la concepción de los cuatro principios genera una serie de contradicciones que desequilibra la práctica bioética, tanto considerados individualmente como en relación unos con otros. El problema que surge con el principio de autonomía al dogmatizarse es que deviene en la prosecución de un exceso de autonomía y de una compartimentación de la información. El exceso de autonomía se traduce en una doble problemática: por un lado, la tecnificación excesiva de la híper-especialización de la medicina que "en la medida en que trata con personas

\footnotetext{
${ }_{17}$ Gafo, Javier. 10 palabras clave en Bioética. Navarra, Editorial Verbo Divino, 1997, p. 32.

18 Gracia, Diego. Procedimientos de decisión en ética clínica, Madrid: Editorial Eudema, 1991.
} 
necesita ver no sólo enfermedades como patologías generales, sino enfermedades de alguien"; ${ }^{19}$ y por otro lado, el desprestigio público de la práctica médica ante esta progresiva deshumanización e instrumentalización de los pacientes (abriendo la puerta a pseudo-conocimientos médicos). Sin un equilibrio en la relación médico-paciente no es posible establecer un diálogo común, sino una imposición entre posturas radicales: la visión de la autonomía del paciente contra la visión de la autonomía del médico.

El problema con la dogmatización del principio de beneficencia es que en él subyace un marcado paternalismo del médico ${ }^{20}$ con respecto al paciente, restringiendo su autonomía para deliberar y tomar decisiones. El paternalismo es "1) la limitación intencionada de la autonomía de una persona por parte de otra 2) cuando la persona que limita autonomía apela exclusivamente a motivos de beneficencia hacia la persona cuya autonomía es limitada". ${ }^{21}$ Del paternalismo ${ }^{22}$ se deriva una de las (falsas) contradicciones de la práctica bioética: ¿principio de autonomía o principio de beneficencia? La actitud del médico no debe ser tanto querer curar al paciente a toda costa, como sí averiguar qué le sucede para prescribir un tratamiento. Una postura inquisitorial subvierte la práctica médica y la convierte en la imposición de un tratamiento sin importar la condición o la calidad de vida ${ }^{23}$ del paciente.

El problema que surge con la dogmatización del principio de no-maleficencia es, al igual que sucedía con el principio de beneficencia, que se ejercer una relación paternal con el enfermo, pero la diferencia entre ambos estriba en la acción que se ejerce, o mejor dicho, la que no ejerce el médico en el paciente: en la beneficencia el médico actúa directa y activamente sobre en paciente, pero en la no-maleficencia el médico, actuando de una forma pasiva (o recurriendo directamente a la inacción), busca no perjudicar al enfermo, evitando infligirle un daño innecesario. La compli-

19 Domingo Moratalla, Tomas. "Bioética hermenéutica", en Bioética: el pluralismo de la fundamentación (Coords. José Jorge Ferrer, Juan Alberto Lecaros Urzúa, et al.), Madrid, Universidad Pontificia de Comillas, 2016, pp. 296-297.

${ }^{20}$ La figura del médico dentro de la práctica médica tiene una importancia ańadida al resto de los implicados: es quien tiene el conocimiento y la habilidad para curar. Por tanto, un cierto paternalismo resulta casi inevitable en el ejercicio de la medicina. Sin embargo, el problema en torno al paternalismo es cuando se despliega de modo ostentoso y abusivo, limitando intencionadamente la independencia del paciente para coaccionar su toma de decisiones.

21 Beauchamp, Tom; y McCullough, Laurence. Ética Médica: las responsabilidades morales de los médicos, Barcelona, Editorial Labor, 1987, p. 98.

22 Se ha especulado mucho acerca del paternalismo médico, apareciendo una importante cantidad de argumentos tanto en contra como a favor, incluso dentro de los argumentos en pro del paternalismo hay quienes abogan por una idea de paternalismo condicionado (paternalismo débil) y quienes abogan por un paternalismo sin restricciones (paternalismo fuerte).

23 Véase, Jonsen, Albert; Siegler, Mark; et al. Ética Clínica: aproximación práctica a la toma de decisiones éticas en la medicina clínica, Barcelona, Editorial Ariel, 2005. 
cación con la no-maleficencia es que se transforma en una práctica para evitar los posibles litigios por mala praxis: no actuando se evita actuar mal.

El problema con el principio de justicia es que formalmente asegura un modelo sanitario universal e imparcial que garantiza la distribución equitativa de los recursos sanitarios, legitimándose en un Estado de derecho. Sin embargo, materialmente, el modelo sanitario ha implementado en los últimos años una serie de reformas que lo ha condenado a una gradual restricción de la calidad en los recursos y los servicios sanitarios públicos. Las consecuencias de este proceso se aprecia en la práctica médica diaria: un nepotismo en el acceso a los recursos y los servicios, y una calidad deficiente en la administración que, por otro lado, afronta la privatización progresiva de diferentes servicios médicos. De esta manera, las condiciones coyunturales (poder adquisitivo, condición social, creencias, sexualidad...) terminan influyendo en los recursos y la calidad del servicio que reciben. Con la dogmatización del principio de justicia la imparcialidad se pierde y la función equitativa se queda en una vana ilusión.

La conjunción teórica de los cuatro principios genera un problema de inconsistencia bioética: no se pueden tomar los principios de forma dogmática porque pierden su condición de prima facie y se convierten en principios morales absolutos, pervirtiendo la práctica médica y deslegitimando la validez y la coherencia de la matriz teórica del enfoque principialista. El modelo bioético predominante, basado en esta aberración principialista, se convierte en un mecanismo de ponderación instrumental de beneficio, coste y riesgo más allá de la práctica médica para conjugar intereses ajenos a la propia medicina.

\section{Una corrección hermenéutica.}

ANTE LAS CARENCIAS PRESENTADAS sucintamente sobre el enfoque principialista, la propuesta que defendemos para un análisis satisfactorio y englobador de los complejos problemas que se presentan contemporáneamente en la práctica médica es apostar por la bioética hermenéutica ${ }^{24}$ como la dimensión general de la bioética donde se integrarían los problemas éticos y las diferentes perspectivas bioéticas (como por ejemplo las éticas feministas, las éticas de la virtud, las éticas del cuidado, y las éticas narrativas), precisamente por su proximidad "a la praxis médica real, a la experiencia del paciente, busca considerar siempre la pluralidad de aspectos en un esfuerzo de globalidad e integración de perspectivas". ${ }^{25}$

\footnotetext{
24 Véase, Domingo Moratalla, Tomás. "Bioética hermenéutica", en Bioética: el pluralismo de la fundamentación, Madrid, Universidad Pontificia de Comillas, 2016, pp. 276-302.

25 Domingo Moratalla, Tomás. "Bioética hermenéutica”, op. cit., p. 298.
} 
La bioética hermenéutica, apoya en las principales características de la ética hermenéutica ${ }^{26}$, emplea, frente a la razón formal y la metodología lineal del enfoque principialista, una suerte de razón narrativa y una metodología circular donde la interpretación y la crítica son las herramientas clave para ampliar el análisis bioético de una manera multidimensional y llegar a comprehender el conflicto ético.

Gracias a la dimensión general que proporcionaría la bioética hermenéutica a la bioética médica, el acceso y la pertinencia del fundamento de la bioética: los conflictos éticos, sería reformulado. De tal manera que el análisis bioético de los casos particulares en conflicto -lejos de la esterilidad del enfoque casuístico tradicional- se dirimirían a través de juicios contingentes y propiamente hermenéuticos (interpretativos) donde, en lugar de apelar al recurso de los principios prima facie, estaríamos hablando de pautas de interacción narrativas que tomarían la forma de máximas morales ${ }^{27}$ mediante las cuales se pretendería alcanzar una a) $\lambda \eta \dot{\theta} \theta \varepsilon ı \alpha$ прфктікń, una verdad práctica -en un sentido aristotélico actualizado-. ${ }^{28}$

De ser todo ello cierto [lo que dice la bioética hermenéutica], la bioética habría dado por fin con el hilo de Ariadna que se permitiría escapar de aplicacionismo, los fundamentalismos, el subjetivismo personalista, y el relativismo cultural, para caminar decididamente hacia la construcción de una ética universal, basada en el ser del hombre, descubierto mediante una aproximación hermenéutica. ${ }^{29}$

La formación en la bioética hermenéutica ayuda a no modificar ni simplificar la realidad conflictiva de la práctica médica porque la "bioética es necesariamente subjetiva y relativa; lo que hace la hermenéutica es subrayarlo, asumirlo y volverlo productivo. Pero esto no quiere decir que tenga que ser subjetivista y relativista" ${ }^{30}$. La vocación educativa de la bioética hermenéutica, la apertura a la

\footnotetext{
${ }^{26}$ Véase, Conill Sancho, Jesús. Ética hermenéutica: crítica desde la facticidad, Madrid, Editorial Tecnos, 2010.

27 Al hablar de «máximas morales» entendemos -siguiendo a Tomás Domingo Moratalla y Lydia Feito Grandeque son "expresiones de sabiduría práctica alcanzada a lo largo del tiempo, puesta a prueba en la experiencia. Son construcciones históricas circunstanciadas, criterios de prudencia que pueden ser revisables, pero que no se han establecido de un modo arbitrario" (Domingo Moratalla, Tomás; y Feito Grande, Lydia. Bioética narrativa, Madrid, Escolar y Mayo Editores, 2013, p. 88).

28 El concepto de «verdad práctica» proviene del pensamiento de Aristóteles, pero no se encuentra abiertamente en ninguna de sus obras, sino que, más bien, se entiende como la consecución práctica de su noción de "prudencia»

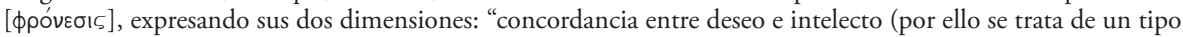
de verdad) y creación de un bien objetivo (por ello es práctica)" (Marcos, Alfredo. Ciencia y acción: una filosofía práctica de la ciencia, México, Fondo de Cultura Económica, 2010, 136).

La actualización de la verdad práctica es el resultado de las sucesivas correcciones realizadas en las experiencias y las vivencias personales para alcanzar un conocimiento narrativo e intersubjetivo sobre la realidad mutable del mundo.

29 García Llerena, Viviana María. De la bioética a la biojurídica: el principialismo y sus alternativas, Granada, Editorial Comares, 2012, p. 151.

30 Domingo Moratalla, Tomas. “Bioética hermenéutica”, op. cit., p. 270.
} 
narración y la comunicación -en un sentido jaspersiano-, ${ }^{31}$ la valoración de las cuestiones culturales, las socio-económicas y las de género; permiten a la bioética hermenéutica acceder al espacio intersubjetivo común que existe entre los seres humanos y dar visibilidad a conflictos éticos silenciados hasta ahora. Por tanto, la bioética hermenéutica se encontraría en constante conexión con los problemas de nuestra época.

31 Véase, Jaspers, Karl. Filosofía (Vol.1), Madrid, Revista de Occidente, 1958; y Jaspers, Karl. Filosofía (Vol. 2), Madrid, Revista de Occidente, 1959. 


\section{Referencias Bibliográficas}

Aristóteles. Ética Nicomáquea. Ética Eudemia, Madrid, Editorial Gredos, 2008.

Beauchamp, Tom; y Childress, James Franklin. Principios de ética biomédica, Barcelona, Editorial Masson, 1999.

Beauchamp, Tom; y McCullough, Laurence. Ética Médica: las responsabilidades morales de los médicos, Barcelona, Editorial Labor, 1987.

Conill Sancho, Jesús. Ética hermenéutica: crítica desde la facticidad, Madrid, Editorial Tecnos, 2010.

Domingo Moratalla, Tomas. "Bioética hermenéutica", en Bioética: el pluralismo de la fundamentación (Coords. José Jorge Ferrer, Juan Alberto Lecaros Urzúa, et al.), Madrid, Universidad Pontificia de Comillas, 2016, pp. 267-302.

Domingo Moratalla, Tomás; y Feito Grande, Lydia. Bioética narrativa, Madrid, Escolar y Mayo Editores, 2013.

Gafo, Javier. 10 palabras clave en Bioética. Navarra, Editorial Verbo Divino, 1997.

García Llerena, Viviana María. De la bioética a la biojurídica: el principialismo y sus alternativas, Granada, Editorial Comares, 2012.

Gómez Ramos, Antonio. "Política sin medios y violencia sin fines: Hannah Arendt y Walter Benjamin sobre la violencia”, en Cuaderno gris, No. 10, 2012, pp. 187-206.

Gracia, Diego. Procedimientos de decisión en ética clínica, Madrid: Editorial Eudema, 1991.

Jaspers, Karl. Filosofía (Vol. 2), Madrid, Revista de Occidente, 1959.

- Filosofia (Vol.1), Madrid, Revista de Occidente, 1958.

Jonsen, Albert. "Casuistry as Methodology in Clinical Ethics", en Theoretical Medicine, 12, 1991, pp. 299-302.

Jonsen, Albert; Siegler, Mark; et al. Ética Clínica: aproximación práctica a la toma de decisiones éticas en la medicina clínica, Barcelona, Editorial Ariel, 2005. 
Marcos, Alfredo. Ciencia y acción: una filosofía práctica de la ciencia, México, Fondo de Cultura Económica, 2010, 136

Moreno, Jonathan. "The end of the great bioethics compromise", en The Hastings Center Report, 35, 2005, pp. 14-15.

Ten Have, Henk. "Bioética: el arte de la interpretación y la deliberación", en Bioética: el estado de la cuestión (Eds. Lydia Feito, Diego Gracia, y Miguel Sánchez), Madrid, Editorial Triacastela, 2011, pp. 155-174.

Wolf, Susan. "Gender and feminism in bioethics", en Feminism and Bioethics: beyond reproduction (Ed. Susan Wolf), Nueva York, Oxford University Press, 1996, pp. 3-43.

DOI: http://dx.doi.org/10.15366/bp2018.18.009

Bajo Palabra. II Época. No18. Pgs: 195-208 